\title{
Rhodiola crenulata extract for prevention of acute mountain sickness: a randomized, double-blind, placebo-controlled, crossover trial
}

Te-Fa Chiu ${ }^{1,2}$, Lisa Li-Chuan Chen ${ }^{1}$, Deng-Huang Su ${ }^{3,4}$, Hsiang-Yun Lo ${ }^{1,2}$, Chung-Hsien Chen ${ }^{5}$, Shih-Hao Wang ${ }^{6,7^{*}}$ and Wei-Lung Chen ${ }^{8,9^{*}}$

\begin{abstract}
Background: Rhodiola crenulata ( $R$. crenulata) is widely used to prevent acute mountain sickness in the Himalayan areas and in Tibet, but no scientific studies have previously examined its effectiveness. We conducted a randomized, double-blind, placebo-controlled crossover study to investigate its efficacy in acute mountain sickness prevention.

Methods: Healthy adult volunteers were randomized to 2 treatment sequences, receiving either $800 \mathrm{mg}$ R. crenulata extract or placebo daily for 7 days before ascent and 2 days during mountaineering, before crossing over to the alternate treatment after a 3-month wash-out period. Participants ascended rapidly from $250 \mathrm{~m}$ to $3421 \mathrm{~m}$ on two separate occasions: December 2010 and April 2011. The primary outcome measure was the incidence of acute mountain sickness, as defined by a Lake Louise score $\geq 3$, with headache and at least one of the symptoms of nausea or vomiting, fatigue, dizziness, or difficulty sleeping.

Results: One hundred and two participants completed the trial. There were no demographic differences between individuals taking Rhodiola-placebo and those taking placebo-Rhodiola. No significant differences in the incidence of acute mountain sickness were found between $R$. crenulata extract and placebo groups (all 60.8\%; adjusted odds ratio $(A O R)=1.02,95 \%$ confidence interval $(C l)=0.69-1.52)$. The incidence of severe acute mountain sickness in Rhodiola extract vs. placebo groups was $35.3 \%$ vs. $29.4 \%$ ( $A O R=1.42,95 \% \mathrm{Cl}=0.90-2.25)$.
\end{abstract}

Conclusions: $R$. crenulata extract was not effective in reducing the incidence or severity of acute mountain sickness as compared to placebo.

Trial registration: ClinicalTrials.gov NCT01536288.

Keywords: Acute mountain sickness, High altitude illness, Rhodiola

\section{Background}

Acute mountain sickness (AMS) occurs in individuals rapidly ascending to high altitude and it usually results in headache, along with anorexia or nausea, fatigue, dizziness, and insomnia [1]. The number of people travelling rapidly to higher altitudes for work or recreation is rising. As a result of improvements in transportation to these high-altitude regions, AMS has become a significant

\footnotetext{
* Correspondence: mountainwangsh@gmail.com; er.mountain.gogo@gmail. com

${ }^{6}$ Department of Emergency Medicine, Taipei Medical University Hospital, Wu-Hsing Street, Taipei, Taiwan

${ }^{8}$ Department of Emergency Medicine, Cathay General Hospital, Sec. 4, Jen-Ai Road, Taipei, Taiwan

Full list of author information is available at the end of the article
}

environmental health issue [2]. The most effective preventive method for AMS-gradual ascent-is usually difficult or impractical for modern international travelers to locations such as Lhasa in Tibet $(3650 \mathrm{~m})$ and La Paz in Bolivia $(3740 \mathrm{~m})$ [3]. The pathophysiology of AMS is not totally understood but it is apparent that administration of prophylactic treatments is helpful. Historically acetazolamide has been used as a gold standard in treatments to prevent malaise symptoms at high altitudes [1,2,4-7]. However, acetazolamide requires a prescription and has side effects such as paresthesia, dysgeusia, and diuresis $[2,8,9]$. Some over-the-counter herbal supplements such as Rhodiola species, Ginkgo biloba and Coca leaf products are widely used [3,5,7,10-17].

\section{Biomed Central}

(C) 2013 Chiu et al.; licensee BioMed Central Ltd. This is an open access article distributed under the terms of the Creative Commons Attribution License (http://creativecommons.org/licenses/by/2.0), which permits unrestricted use, distribution, and reproduction in any medium, provided the original work is properly cited. 
Genus Rhodiola (family Crassulaceae) has been a valuable medical plant in European countries for thousands of years $[10,18,19]$. There are more than ninety species worldwide and they are well known for their high antioxidant activities. Different species of Rhodiola differ in their content of bioactive components and medicinal use in indigenous regions. To date, most studies focus on the efficacy of Rhodiola rosea in treating physical and mental fatigue. Of all the known Rhodiola species, Rhodiola crenulata ( $R$. crenulata) is particularly described in the Pharmacopoeia of China [16] and is considered as the highest in quality. Also, it has been used for treating acute mountain sickness in Tibet since ancient times [20].

Pulmonary alveolar hypoxia, a common phenomenon for non-acclimatized individuals who abruptly relocate to a high altitude, is thought to contribute to the impaired trans-alveolar fluid transport. The excessive fluid that subsequently accumulates in alveoli exaggerates alveolar hypoxia and obstructs the gas exchange process, leading to the pathological progression of high altitude pulmonary edema, the most lethal form of high-altitude illnesses $[21,22]$. In a rodent model, $R$. crenulata extract exhibited a high antioxidant activity and attenuated pulmonary edema induced by hypobaric hypoxia [20]. Recent studies have shown that $\mathrm{Na} / \mathrm{K}$-ATPase plays a key role in alveolar fluid clearance. Both inhibition and knockdown of $\mathrm{Na} / \mathrm{K}$ ATPase expression significantly reduced the alveolar fluid clearance in rodent models $[23,24]$. Two bioactive components of $R$. crenulata, salidroside and tyrosol, were shown to hold antioxidant, anti-depression, anti-fatigue, and antiinflammatory activities [25-30]. Therefore, the capabilities of $R$. crenulata in preventing the hypoxia-mediated $\mathrm{Na}$ / K-ATPase endocytosis and maintaining the integrity of alveolar-capillary barrier and pulmonary sodium transport might be the underlying mechanisms of its effect against pulmonary edema in rodent models [31].

Although $R$. crenulata is widely used to prevent AMS in the Himalayan areas and in Tibet [20,32], but no scientific studies have examined its effectiveness in humans. We conducted a controlled study to investigate the efficacy of $R$. crenulata in AMS prevention. In the clinical trials for Rhodiola, most studies were conducted to investigate whether Rhodiola could improve the endurance exercise performance or protect against fatigue. The daily dosage of Rhodiola extract tested was mostly under $500 \mathrm{mg}$ (ranged between 100-972 mg) [15,18,19]. Regarding the duration of prophylactic medication for AMS, chemical drugs such as acetazolamide could elicit a quick response and were usually administered on the day before or prior to the ascent. In the trials on AMS prevention using prophylactic herbal medication, Ginkgo biloba was the one mostly studied. It was often administered for one to five days prior to the ascent $[3,11-13]$. Considering the onset of AMS after ascent and the compliance of prophylactic medication, a high dose of $R$. crenulata (800 mg daily) was chosen in this study and the subjects were administered with $R$. crenulata for seven days prior to the ascent.

\section{Methods}

\section{Ethics statement}

This study has been approved by the Institutional Review Board (IRB) of Chang Gung Medical Foundation, Linkou Medical Center, Taoyuan, Taiwan. The IRB approved number is "99-3057C" and the topic is titled "Can Rhodiola Crenulata Intake Improve Oxygen Saturation and Decrease the Incidence of Acute Mountain Sickness". Subjects were given written information and a verbal explanation concerning the study prior to obtaining the written informed consent for their participation.

\section{Subjects}

We included local Chinese adults aged 23 to 55 years who resided principally at an elevation of $250 \mathrm{~m}$ or lower. We excluded those who (1) would not complete the study protocol of two 9-day treatment courses; (2) had prophylactic medication or herbs one month before each ascent (including acetazolamide, sildenafil, tadalafil, dexamethasone, nifedipine, Rhodiola species, Ginkgo biloba, Eleuthero Root, Salvia miltiorrhiza, and sea buckthorn); (3) change in altitude of residence for more than $200 \mathrm{~m}$ between ascents; (4) had additional physical training or were scheduled to gain or lose weight; (5) had altitude exposure above $2500 \mathrm{~m}$ within three months prior to each ascent; (6) had any history of chronic obstructive pulmonary disease, heart failure, cerebral neoplasm, mania, renal or hepatic insufficiency; or (7) were pregnant or intended to become pregnant during the 3-month study period. After baseline assessment, the subjects were randomly assigned into either sequence.

\section{Study design, randomization and blinding}

This study was a randomized, double-blind, placebocontrolled and crossover trial. Subjects were randomized to 2 treatment sequences, receiving either the $R$. crenulata or the placebo, and then crossed over to the alternate treatment after a 3 month wash-out period. Random numbers were generated by using the computer, using block randomization with a block size of 2 or 4 . The random numbers were placed in sealed envelopes, and a serial number was assigned to each envelope according to the sequence of allocation of the randomized number. Each envelope was then opened sequentially, according to the admission sequence of the participants at the study center. The number inside the envelope determined the treatment sequence that each participant was allocated to (Rhodiolaplacebo or placebo-Rhodiola). Both investigators and participants were blinded. One investigator, who was not responsible for any assessment, enrolled all participants 
and allocated them to treatment. Blinding was maintained until the data analysis was complete.

\section{Medications}

Commercial pharmaceutical grade $R$. crenulata and placebo were packed by Kaiser Pharmaceutical \& Biotanico (Tainan, Taiwan). Both were pink soft gelatin capsules $(400 \mathrm{mg} /$ capsule) in identical containers and there were no differences in the taste or smell of the capsules. The standardized $R$. crenulata extract (with $2.38 \%$ salidroside and $0.44 \%$ $p$-tyrosol) was manufactured from the same batch of raw materials $[33,34]$. The origin of the herb was authenticated by microscopic identification and sequence analysis of the internal transcribed spacer regions. The material was examined for microorganisms, heavy metals, and pesticide according to the accepted standards (good manufacturing practice, GMP) of Taiwan. Each participant received a high dose $(800 \mathrm{mg}$ ) of $R$. crenulata or placebo $(800 \mathrm{mg}$ ) daily for 9 days. Beginning 7 days before an ascent, participants were required to inform the investigators by email or telephone every day about the study capsule supply or any adverse effects. If the investigators were not contacted before noon, they would follow up by phone to obtain this information. Moreover, the participants were requested to take capsules every morning of their 2-day mountaineering trip.

\section{Ascent}

To control the ascent rate, participants were transported by bus from an altitude of $250 \mathrm{~m}$ to $3100 \mathrm{~m}$ (the training camp) within $4 \mathrm{~h}$ (Figure 1); they rested for $2 \mathrm{~h}$, hiked $1 \mathrm{~km}$ to the East Peak $\left(3421 \mathrm{~m}, 24^{\circ} \mathrm{N} 121^{\circ} \mathrm{E}\right)$ of Hehuan
Mountain, Taiwan within $1.5 \mathrm{~h}$, rested $30 \mathrm{~min}$, and descended back to the training camp $(3100 \mathrm{~m})$, where they remained overnight. On the second day, all participants were transported by bus to the entrance $(3200 \mathrm{~m})$ of the main peak of Hehuan Mountain, and they went hiking along the easy trekking path to the peak (3416 m). After that, they returned to the entrance of the main peak and traveled back to Linkou by bus. There were 10 checkpoints for assessment, namely, Linkou (250 m), Cingjing Farm $(1743 \mathrm{~m})$, Yuanfeng parking lot $(2756 \mathrm{~m})$, training camp $(3100 \mathrm{~m})$, the East Peak $(3421 \mathrm{~m})$ of Hehuan Mountain, training camp in the evening and morning, the Main Peak (3416 m) of Hehuan Mountain, Cingjing Farm, and Linkou. During the mountaineering trips, the following variables were controlled: rate of ascent, path, food type, and sleep altitude/environment. The training camp could not accommodate all subjects at once. Thus, the participants were divided into 2 groups in each period, and they ascended separately in less than 10 days apart. The usual snow season of the Hehuan Mountain is between early January and early March. We chose early and midDecember 2010, late March, and early April 2011 as our 4 mountaineering dates. The atmospheric pressure and temperature were recorded at each checkpoint during every trip.

\section{Measurements}

The Lake Louise scoring system (LLS) - a simple and widely accepted tool developed by the Lake Louise Consensus Group [1,35] —was used to assess the AMS grade. The LLS rates 5 symptoms (headache, gastrointestinal symptoms such as nausea and vomiting, fatigue and/or

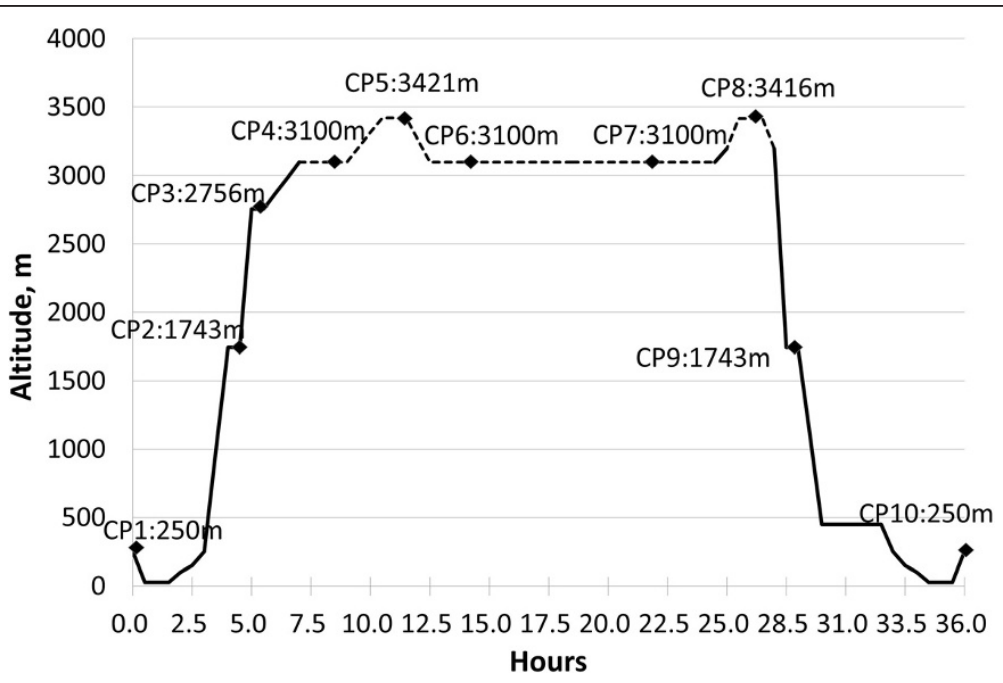

Figure 1 Mountaineering schedule. Total of 10 checkpoints (CPs): CP 1, Linkou (250 m); 2, Cingjing Farm (1743 m); 3, Yuanfeng parking lot (2756 m); 4, training camp (3100 m) at noon; 5, East Peak (3421 m) of Hehuan Mountain; 6, training camp (evening); 7, training camp (next morning); 8, Main Peak (3416 m) of Hehuan Mountain; 9, Cingjing Farm and 10, Linkou. Solid line indicates that participants were transported by bus. 
weakness, dizziness and/or light-headedness, and difficulty sleeping) on a self-report questionnaire, with each item graded on a scale from 0 to 3 . Thus, the LLS scores can range from 0 (no symptoms or signs) to 15 (the worst rating on each symptom) $[12,13,35]$.

The AMS grade, Lake Louise self-report questionnaire, and pulse oximetry $\left(\mathrm{SpO}_{2}, \mathrm{NPB} 40\right.$, Nellcor, Pleasanton, CA, USA) were completed at 10 checkpoints along the route. Four emergency physicians, familiar with the management of AMS, took care of any discomfort of the participants during the whole course of mountaineering. Participants were not permitted to self-treat their AMS symptoms. Instead, they were told in advance that investigators would provide oral acetaminophen $(500 \mathrm{mg}$ ) or ibuprofen (400 $\mathrm{mg}$ ) for headache, meclizine $(25 \mathrm{mg}$ ) for dizziness or intramuscular prochlorperazine $(5 \mathrm{mg})$ for nausea and/or vomiting.

All subjects had their own mountaineering health passport. It had a list of symptoms related to AMS, advice and precautions to take during mountaineering, a place to record pulse oximetry values, and the Lake Louise questionnaire (to be filled out at each checkpoint). It was also used to record clinical problems and treatment.

\section{Outcomes}

The predetermined primary outcome was the incidence of AMS. It was evaluated at checkpoints 3 to 8 (altitude above $2500 \mathrm{~m}$ ) or at any time of discomfort after ascent. AMS was defined as LLS score $\geq 3$ with headache and at least one of the symptoms of nausea or vomiting, fatigue, dizziness, or difficulty sleeping. Predetermined secondary outcomes included incidence of severe acute mountain sickness (LLS score $\geq 5$ ), incidence of headache and severe headache (defined as a headache score of 2 or 3 on the headache item of LLS), oxygen saturation by pulse oximetry $\left(\mathrm{SpO}_{2}\right)$ at checkpoint $4(3100 \mathrm{~m})$ and its difference between altitudes $250 \mathrm{~m}$ and $3100 \mathrm{~m}\left(\Delta \mathrm{SpO}_{2}: \mathrm{SpO}_{2}\right.$ measured at checkpoint 1 minus $\mathrm{SpO}_{2}$ at checkpoint 4).

\section{Sample size}

The incidence of AMS in placebo group was hypothesized as $36 \%$ according to our previous survey [36]. A relative risk reduction of $30 \%$ was considered the clinically significant effect in this type of treatment. According to the study design, assuming the absence of carry-over or period effects, a sample size of 133 participants was deemed sufficient to provide a power of $80 \%$ with a two-sided alpha level of 0.1 and a probability of discordant pairs of 0.7 to detect an odds ratio for the incidence of AMS of 0.59 between the Rhodiola group and the placebo group.

\section{Statistical methods}

Baseline characteristics are presented as mean \pm SD (standard deviation) or counts (percentages), as appropriate. For each variable, participants were grouped according to sequence (Rhodiola-placebo or placebo-Rhodiola) to make the baseline comparison. Comparisons between the two sequences were analyzed by the two-sample independent $t$ test for continuous variables and Chi-square test/ Fisher exact test for categorical variables. Carry-over effects and period effects were assessed using the generalized linear models with generalized estimating equations, assuming a logit link function and an unstructured correlation.

If neither a period nor a carry-over effect occurred, paired $t$ test or McNemar test was used to test the treatment effect. Otherwise, the generalized linear models with generalized estimating equations were used to analyze the treatment effect, assuming a logit link function and an unstructured correlation for binary outcomes or an identity link function and an unstructured correlation for continuous outcomes, and assuming adjustment for period and carry-over effects in the model. All statistical assessments were evaluated at a two-sided significance level of 0.05 . Analyses were performed with SAS software package, version 9.2 (SAS Institute Inc., Cary, NC, USA).

\section{Results}

\section{Participants}

All 125 participants were recruited to the study in November 2010, and they made two separate trips to Hehuan Mountain: one in early or mid-December 2010 and one in April 2011 or early May. On the second trip, the ascent of one half of the participants was terminated midway $(1740 \mathrm{~m})$ because of an unexpected snow storm in late March 2011. Another trip was rescheduled to early May 2011. The trial flow diagram is shown in Figure 2. In all, 23 participants withdrew: 5 from the Rhodiolaplacebo sequence before the first ascent, 3 from the placebo-Rhodiola sequence before the first ascent, 2 from the Rhodiola-placebo sequence during the first ascent, 8 from the Rhodiola-placebo sequence before the second ascent, and 5 from the placebo-Rhodiola sequence before the second ascent. The reasons for withdrawal included interim business trips $(\mathrm{n}=4)$; infections before ascent ( $\mathrm{n}=4$, 3 with upper respiratory tract infection, 1 with leg cellulitis); illness of a girlfriend $(n=1)$; missed departure due to oversleeping $(n=1)$; and postponement of the second trip ( $n=6$ because of work shift problems and 5 due to scheduled travel abroad). One subject in the Rhodiola-placebo sequence developed severe vertigo and vomiting at $7 \mathrm{~h}$ after ascent during the first trip and was urgently evacuated. Her spouse who also was in the Rhodiola-placebo sequence descended with her. The full study protocol was completed by 102 participants. All participants were exposed to the same temperatures, as no statistically significant differences were found between the four ascents (Table 1). 


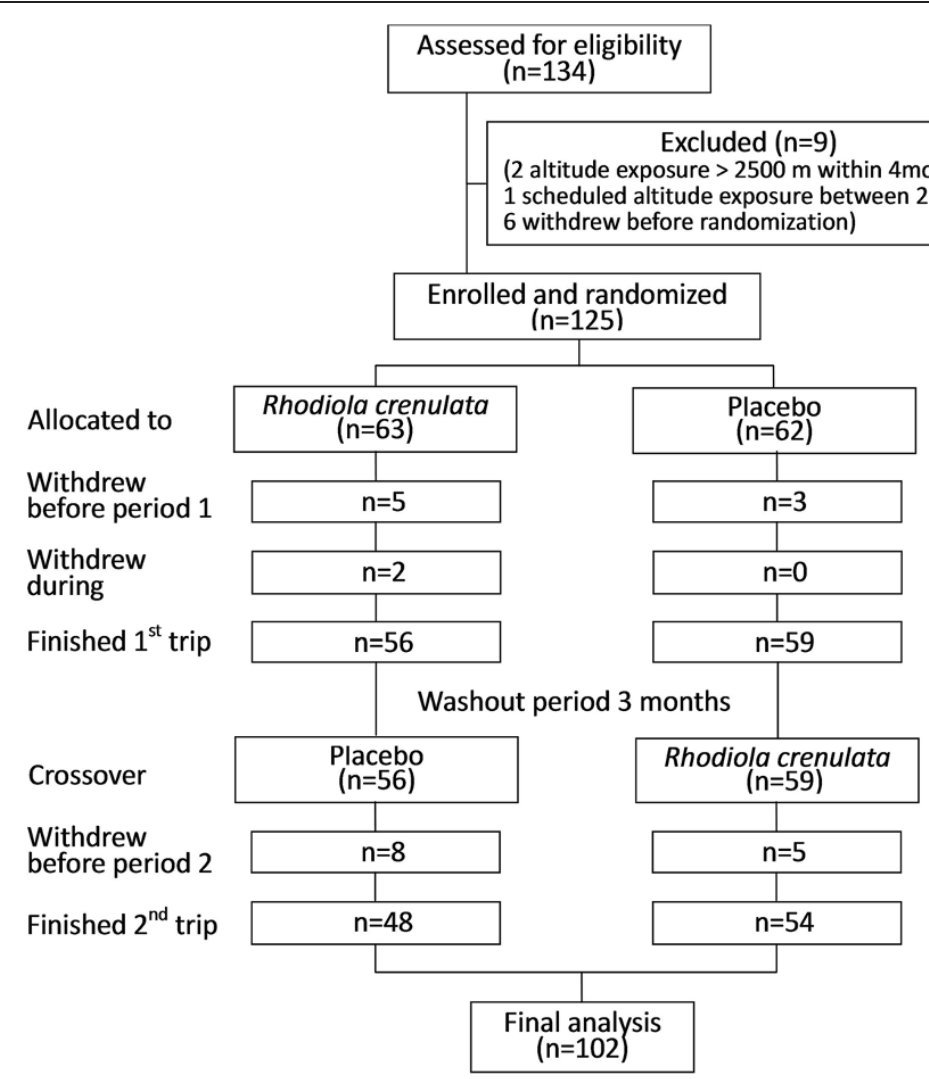

Figure 2 Participant flow diagram.

\section{Baseline characteristics}

None of the participants (54 women, $48 \mathrm{men}$; average age, $36.1 \pm 10.2$ years) went mountaineering regularly, but none avoided outdoor sports. Participants in both sequences (Table 2), Rhodiola-placebo and placebo-Rhodiola, had similar baseline characteristics (gender, age, body mass index, blood pressure, blood oxygen content, heart rate, mountaineering experience, and AMS history before first period; all $P>0.05$ ).

\section{Period effect and carry-over effect}

There was a significant difference in the incidence of AMS between period $2(55.9 \% ; 57 / 102)$ and period 1 (65.7\%; 67/102) (odds ratio: 0.66, 95\% confidence interval:

Table 1 Ambient temperatures during the four ascents

\begin{tabular}{lcccc}
\hline \multirow{2}{*}{ Ascents } & \multicolumn{3}{c}{ Temperature $\left({ }^{\circ} \mathbf{C}\right)$} & \multirow{2}{*}{$\boldsymbol{P}$ value } \\
\cline { 2 - 4 } & Checkpoint 5 & Checkpoint 6 & Checkpoint 7 & \\
\hline Dec. 03, 2010 & 8 & 10 & 9 & 0.060 \\
Dec. 12, 2010 & 9 & 7 & 7 & \\
Apr. 08, 2011 & 9 & 12 & 12 & \\
May 03, 2011 & 10 & 14 & 12 & \\
\hline
\end{tabular}

Comparison of temperature among the four ascents was assessed by Kruskal-Wallis Test.
$0.45 \sim 0.98)$. A period effect $(P=0.040)$ was observed for the primary outcome, but no carry-over effect was found $(P=0.877)$.

\section{Major outcome measures}

The incidence of AMS was the same among participants who received Rhodiola extract or placebo (all 62/102, $60.8 \%$ ). Furthermore, the AMS incidences in the Rhodiola and placebo groups were $66.7 \%$ and $64.8 \%$ in period 1 , compared with $55.6 \%$ and $56.3 \%$ in period 2 , respectively (Figure 3). After adjusting for the period effect, the risk of AMS in participants taking Rhodiola was 1.03 times that of participants taking placebo, and no significant difference was found between the Rhodiola and placebo groups (95\% confidence interval: 0.69-1.52; Table 3). About half of the participants (50/102) had trip 2 rescheduled because of a snowstorm, meaning that those taking Rhodiola had double the planned dosage. Even for these participants, the AMS incidence in the Rhodiola group (56.0\%, 14/25) was not reduced compared to the placebo group $(48.0 \%, 12 / 25)$.

After adjustment for period effect, between-group differences in the incidence of severe AMS, headache, severe headache, $\mathrm{SpO}_{2}$ level and pulse rate at checkpoint 
Table 2 Baseline characteristics

\begin{tabular}{|c|c|c|c|}
\hline \multicolumn{4}{|c|}{ Treatment sequences } \\
\hline Characteristic & Rhodiola - placebo & Placebo - Rhodiola & $P$ value \\
\hline & $(\mathrm{N}=48)$ & $(N=54)$ & \\
\hline Male & $23(47.9)$ & $27(50.0)$ & 0.83 \\
\hline Age, y & $35.8 \pm 10.0$ & $36.3 \pm 10.4$ & 0.84 \\
\hline $\mathrm{BMI}, \mathrm{kg} / \mathrm{m}^{2}$ & $22.6 \pm 2.9$ & $23.4 \pm 3.0$ & 0.17 \\
\hline $\mathrm{SpO}_{2}^{*}, \%$ & $98.8 \pm 0.9$ & $98.9 \pm 1.0$ & 0.60 \\
\hline Heart rate, beats/min & $72.5 \pm 8.9$ & $72.2 \pm 9.6$ & 0.89 \\
\hline Altitude of residence, $\mathrm{m}$ & $151.7 \pm 105.9$ & $152.7 \pm 105.9$ & 0.94 \\
\hline History of mountaineering above 3000 m & & & 0.18 \\
\hline Never & $23(47.9)$ & $36(66.7)$ & \\
\hline$<10$ mountains & $20(41.7)$ & $16(29.6)$ & \\
\hline$>10$ mountains & $5(10.4)$ & $2(3.7)$ & \\
\hline History of AMS & $7(14.6)$ & $5(9.3)$ & 0.54 \\
\hline
\end{tabular}

Continuous variables are presented as mean \pm standard deviation, and categorical variables are presented as count (percentage).

BMI, Body mass index.

${ }^{*} \mathrm{SpO}_{2}$, Oxygen saturation by pulse oximetry at an altitude of $250 \mathrm{~m}$. Heart rate and other demographics were measured during the participant conference before randomization.

AMS, Acute mountain sickness.

4, and $\Delta \mathrm{SpO}_{2}$ level were not significant (all $P>0.05$ ) (Table 3).

\section{Other outcome measures and adverse events}

There were no significant differences between Rhodiola groups and placebo groups for the comparison of the worst AMS symptom scores and highest LLS scores in the interval between checkpoints 3 and 8 (all $P>0.05$ ) (Table 3). The number of subjects requesting medication for headache/dizziness/nausea (vomiting) was about the same in both groups (36/20/4 vs. 35/20/3 for Rhodiola and placebo respectively).

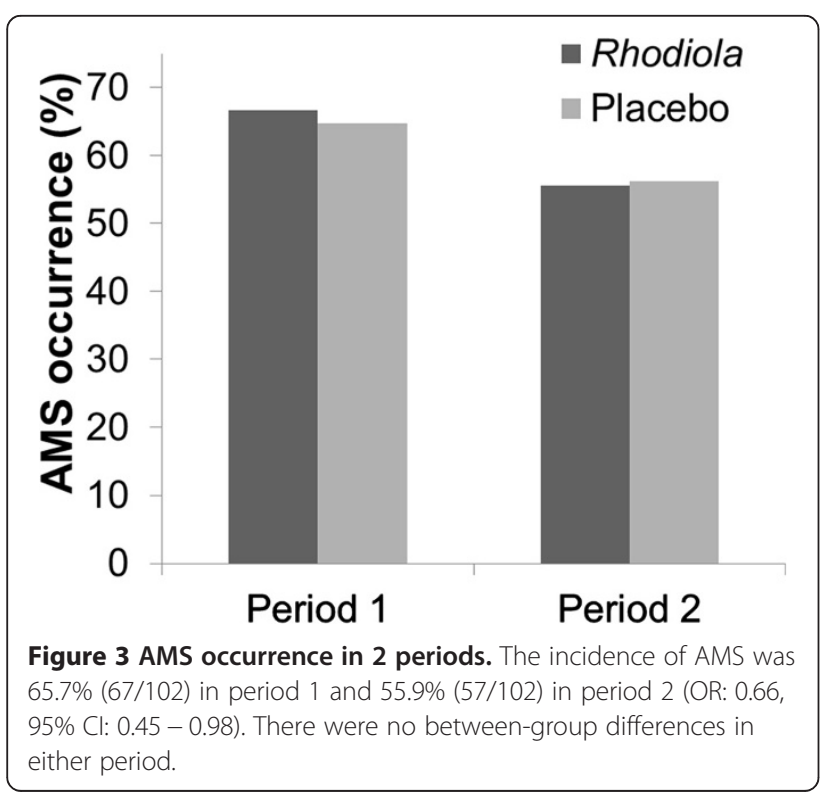

During the 7-day period prior to ascent, adverse events were rare. All were rare, mild and lasted less than two days; therefore, no participant needed to stop taking the study drugs prior to ascent (Table 4).

\section{Sensitivity analysis}

In total, there were fifteen subjects who attended mountain climbing during period 1 but were absent during period 2 (Figure 2). To evaluate the impact of dropout on the efficacy for the Rhodiola arm compared to the placebo arm, two scenario analyses were carried out in order to fill in the missing values for AMS occurrence. In the first scenario, the missing values were assumed to be in favor of Rhodiola in preventing AMS. The odds ratio was 0.88 and $95 \%$ confidence interval contains $1(0.6-1.3, P=0.49)$. In the second scenario which is in favor of the placebo in preventing AMS, and odds ratio was 1.4 and 95\% confidence interval contains $1(0.94-2.11, P=0.10)$. In either scenario, Rhodiola was not effective in preventing AMS (Figure 4).

\section{Assessment of blinding}

After the second trip, all participants were asked to guess the trip in which they had taken Rhodiola; 49\% (50/102) guessed correctly, indicating that the participants identified the treatment period during which they received Rhodiola or placebo only by chance.

\section{Discussion}

$R$. crenulata extract was not effective in reducing the incidence or severity of AMS when compared with placebo and failed to show a protective benefit for any outcome 
Table 3 Outcomes

\begin{tabular}{|c|c|c|c|c|}
\hline Outcome & $\begin{array}{c}\text { Rhodiola } \\
(\mathrm{N}=102)\end{array}$ & $\begin{array}{l}\text { Placebo } \\
(\mathrm{N}=102)\end{array}$ & $\begin{array}{l}\text { Odds ratio/difference } \\
\qquad(95 \% \mathrm{Cl})\end{array}$ & $\begin{array}{c}\text { Adjusted } \\
P \text { value }\end{array}$ \\
\hline \multicolumn{5}{|l|}{ Primary endpoint } \\
\hline $\mathrm{AMS}^{\mathrm{a}}$ & $62(60.8)$ & $62(60.8)$ & $1.02(0.69,1.52)^{+\dagger}$ & 0.90 \\
\hline \multicolumn{5}{|l|}{ Secondary endpoint } \\
\hline Severe $\mathrm{AMS}^{\mathrm{b}}$ & $36(35.3)$ & $30(29.4)$ & $1.42(0.90,2.25)^{+\dagger}$ & 0.13 \\
\hline Headache & $81(79.4)$ & $78(77.5)$ & $1.17(0.75,1.83)^{+\dagger}$ & 0.48 \\
\hline Severe headache ${ }^{c}$ & $33(32.4)$ & $30(29.4)$ & $1.16(0.71,1.89)^{t+}$ & 0.55 \\
\hline $\mathrm{SpO}_{2}, \%^{\mathrm{d}}$ & $88.6 \pm 3.9$ & $88.6 \pm 4.3$ & $-0.13(-0.93,0.66)^{f \neq}$ & 0.74 \\
\hline$\Delta \mathrm{SpO}_{2}, \%$ & $9.6 \pm 3.8$ & $9.5 \pm 4.2$ & $0.16(-0.65,0.97)^{\text {执 }}$ & 0.70 \\
\hline Pulse rate, $/ \mathrm{min}^{\mathrm{e}}$ & $99.2 \pm 14.6$ & $99.8 \pm 14.2$ & $-0.30(-2.61,2.02)^{\text {执 }}$ & 0.80 \\
\hline \multicolumn{5}{|c|}{ Other clinical measures } \\
\hline \multicolumn{5}{|c|}{ AMS symptoms score ${ }^{f}$} \\
\hline Headache & $1.17 \pm 0.81$ & $1.13 \pm 0.83$ & $0.05(-0.10,0.20)^{\text {抽 }}$ & 0.50 \\
\hline Dizziness & $0.87 \pm 0.82$ & $0.79 \pm 0.79$ & $0.09(-0.06,0.25)^{\text {护 }}$ & 0.29 \\
\hline Weakness & $0.94 \pm 0.81$ & $0.99 \pm 0.84$ & $-0.02(-0.17,0.13)^{f \neq}$ & 0.81 \\
\hline Vomiting & $0.52 \pm 0.70$ & $0.41 \pm 0.62$ & $0.12(-0.01,0.25)^{\text {执 }}$ & 0.08 \\
\hline Sleep & $1.45 \pm 0.99$ & $1.55 \pm 0.96$ & $-0.07(-0.29,0.14)^{\text {fキ }}$ & 0.49 \\
\hline LLS score & $3.84 \pm 2.49$ & $3.77 \pm 2.54$ & $0.19(-0.27,0.55)^{\neq \neq}$ & 0.51 \\
\hline
\end{tabular}

For each outcome, data were grouped into two datasets (one for Rhodiola and one for placebo) to create a data summary. Categorical outcomes are presented as count (percentage), and continuous outcomes as mean \pm standard deviation. The treatment effect is presented in the column "Odds ratio/difference $(95 \% \mathrm{Cl})$ ". Odds ratio is used for categorical outcomes and defined as Rhodiola versus placebo, while difference is used for continuous outcomes and defined as Rhodiola minus placebo. The generalized linear models with generalized estimating equations method, assuming logit/ identity link as appropriate and unstructured correlation, was used to obtain the results after adjustment of period effects.

${ }^{a}$ Lake Louise score (LLS score) $\geq 3$ with headache and at least one other symptom was defined as AMS. At each period, occurrence of AMS during checkpoints 3 to 8 was defined as AMS.

${ }^{b}$ AMS and LLS score $\geq 5$.

' Defined as headache score of $>1$ on the headache item of LLS (ascending scale of $0-3$ for severity).

d $\mathrm{SpO}_{2}$, oxygen saturation by pulse oximetry $\left(\mathrm{SpO}_{2}\right)$ at checkpoint $4(3100 \mathrm{~m})$.

$\Delta \mathrm{SpO}_{2}$, the difference between checkpoints 1 and $4\left(\mathrm{SpO}_{2}\right.$ at checkpoint $1-\mathrm{SpO}_{2}$ at checkpoint 4$)$.

e Pulse rate was measured at checkpoint 4 (3100 m).

${ }^{f}$ All symptoms in AMS are defined as the worst score in the interval between checkpoints 3 and 8 .

†† odds ratio.

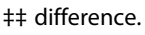

Table 4 Adverse events in groups receiving prophylactic agents for AMS

\begin{tabular}{lcc}
\hline Adverse event & Rhodiola $(\mathbf{n}=\mathbf{1 0 2})$ & Placebo $(\mathbf{n}=\mathbf{1 0 2})$ \\
\hline Difficulty in falling asleep & $1(0.98)$ & $1(0.98)$ \\
Light sleep & $3(2.94)$ & $1(0.98)$ \\
Dizziness & $2(1.96)$ & $0(0.00)$ \\
Drowsiness & $2(1.96)$ & $0(0.00)$ \\
Pruritus & $1(0.98)$ & $1(0.98)$ \\
Dry hand & $1(0.98)$ & $0(0.00)$ \\
Abdominal distension & $1(0.98)$ & $0(0.00)$ \\
General soreness & $1(0.98)$ & $0(0.00)$ \\
Dry mouth & $0(0.00)$ & $3(2.94)$ \\
Headache & $0(0.00)$ & $2(1.96)$ \\
Palpitation & $0(0.00)$ & $2(1.96)$ \\
Flushed face & $0(0.00)$ & $1(0.98)$ \\
Increased urination & $0(0.00)$ & $1(0.98)$ \\
\hline
\end{tabular}

Data are presented as count (\%). measure examined. Statistical analyses showed that there was a period effect but no carry-over effect. AMS occurred significantly less frequently on the second mountaineering trip $(65.7 \%$ vs. $55.9 \% ; P=0.040)$, regardless of treatment. Since most participants $(58 \%)$ had no highaltitude mountaineering experience prior to the first trip, the first trip experience helped them adapt to conditions on the second trip in period 2. Inadequate high-altitude mountaineering experience was proven to be an independent risk factor for AMS [36-39]. In Gaillard's study, the understanding and awareness of AMS among trekkers reduced the incidence of AMS [40]. Also, Vardy et al. suggested that AMS is less likely to take place with a correct awareness of its symptoms and prevention [41]. In our study, the participants filled the Lake Louise self-report questionnaire at 10 checkpoints during mountaineering, so they had a comprehensive awareness of high altitude experience and AMS symptoms after the first period of mountaineering. They also shared experiences and 


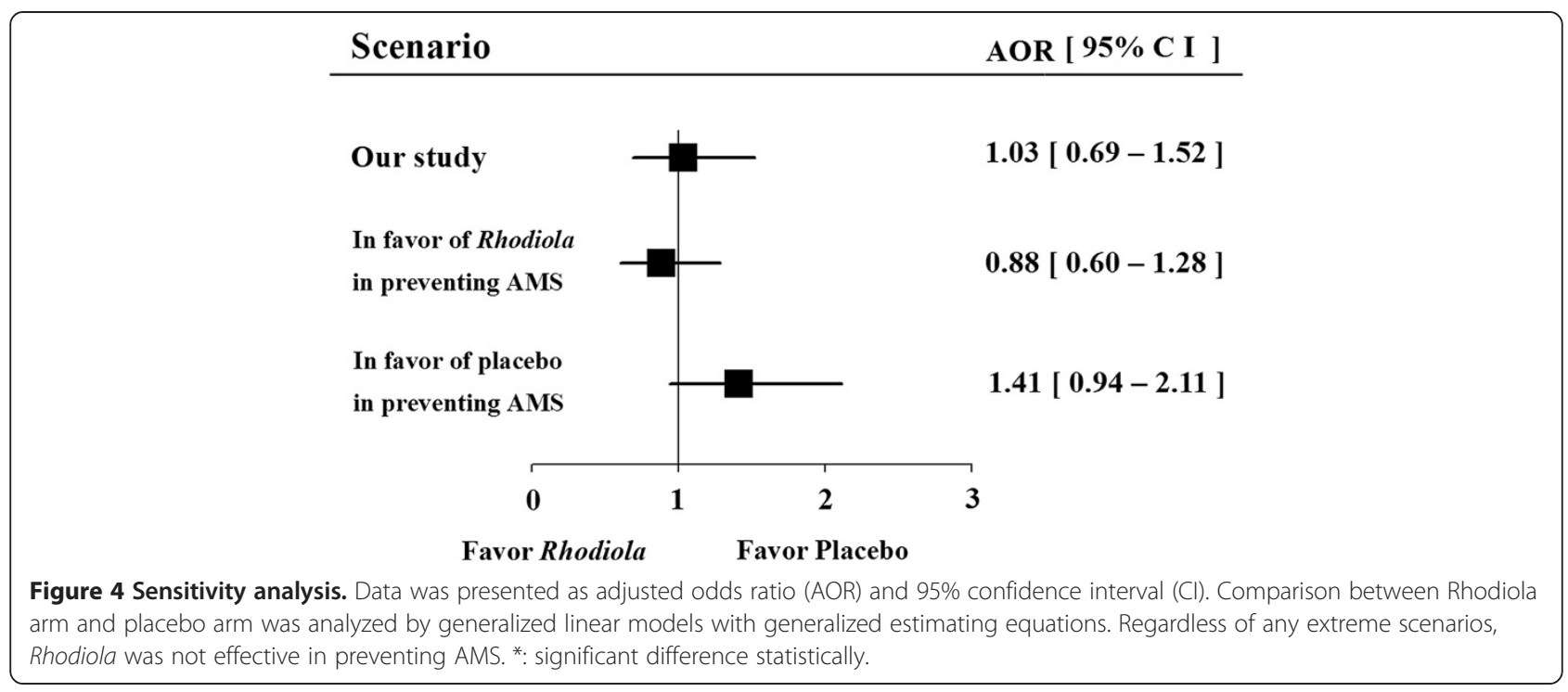

methods on AMS prevention, such as avoiding high impact activity at high altitude. Therefore, the participants had a significantly superior awareness of symptoms and prevention for AMS in the second period than in the first period of mountaineering. Also, no period effect was observed among the experienced participants in our study. Thus, this phenomenon could be classified as a learning effect. The weather, warmer during period 2, could be the other explanation for the period effect. Our trial was designed conservatively to avoid any possible bias against $R$. crenulata: we used a high dose of this agent, a longer period of drug preloading, and a steep ascent profile from the sea level. During the mountaineering trips, the following variables were controlled: rate of ascent, path, food type, and sleep altitude/environment.

In the present study, the environmental and medical resources available to study AMS were limited. We designed our study as a crossover trial for a key reason. Because each participant serves as his or her own control, it skillfully controls for the inherent individual susceptibility to AMS. Each person has his or her own personal tolerance for altitude change, and consequently, we calculated the percentage change of outcomes from his or her baseline to evaluate the effect of intervention or association with possible factors in parallel design. Using crossover design enabled us to measure the effect of intervention exactly, regardless of individual susceptibility. This design also had the benefits of increasing statistical power, given the limited sample size, and efficient control of the influence of confounding covariates.

Our study had several limitations. First, only 102 of the original 125 participants completed both trips. However, the statistical analyses were still adequately powered for three reasons: (1) the incidence of AMS was higher than what we had hypothesized using data generated in our previous survey [36]. This was because most of our participants were inexperienced mountaineers, and their rate of controlled ascent was significantly higher than that in the previous study ( $4 \mathrm{~h}$ vs. $1-2$ days). The participants were requested not to take acetazolamide or other possible prophylactic medications used by trekkers before mountaineering in previous surveys. (2) The sample size was estimated using the assumption that Rhodiola would reduce the incidence of AMS by $11 \%$ ( $36 \%$ to $25 \%$ ) compared to placebo, but the results showed no difference between taking Rhodiola and placebo. Based on the data obtained in the present study, a sample of infinite size would be required to prove that Rhodiola had a significant prophylactic effect. (3) The sensitivity analysis showed that Rhodiola was not effective in preventing AMS regardless of the scenarios proposed (Figure 4). The second limitation is that half of the participants had a rescheduled second trip and they therefore took the study medication (or placebo) for 7 days before ascent and then on the first morning of ascent, for 8 days. Although they did not reach an altitude where AMS might be expected, they took study medication for 8 out of 9 days, and then they began medication once again 4 weeks later. Although these participants took double dosage of Rhodiola, their AMS incidence was not reduced. The third limitation is that the Rhodiola extract purified from the rhizome of $R$. crenulata was used to assure high concentration of active ingredients $(2.38 \%$ salidroside and $0.44 \% p$-tyrosol) in this study. However, the active ingredients for preventing AMS could have been lost during extraction and purification. Many Chinese tourists drink a decoction of Rhodiola rhizome to prevent AMS. The results of the present study do not exclude the possibility that this $R$. crenulata decoction may have a preventive effect on AMS. Last, only one dosage and one Rhodiola species were tested. Higher dosage or extracts 
from other species, such as Rhodiola rosea, Rhodiola kirilowii, Rhodiola sachalinensis, and Rhodiola algida, might be effective.

Studies have investigated different pharmacologic interventions to prevent AMS. Acetazolamide is effective for the prevention of AMS but it may be associated with paresthesias. Sumatriptan and gabapentin are beneficial but require further study [5]. Antioxidants magnesium, Ginkgo biloba and Coca leaf products were not efficacious $[5,17]$. In the Himalayan region, China, and Andean region of South America, using traditional applications of herbs for anti-high altitude illness is more common than using doctor prescription. For example, Coca leaf products were used more often among $62.8 \%$ of the surveyed travelers than acetazolamide prophylaxis (16.6\%) for prevention, or other non-pharmacologic measures [17]. Similar in China, 7\% of 247 Chinese travelers on Qinghai-Tibet railroad took Rhodiola as a prophylaxis for AMS, but none used acetazolamide or dexamethasone, except one who had asthma [32]. It was shown that coca leaf products did not prevent AMS, but increased the chance of AMS occurrence. Therefore, complementary and alternative medicines are omnipresent and popular, and should not be dismissed as "non-medications".

Our study is the first randomized, double-blind and controlled study of $R$. crenulata extract for AMS prophylaxis in humans though the current data does not demonstrate any prophylactic effects. Further studies will be needed to demonstrate the efficacy of $R$. crenulata decoction or other Rhodiola species in AMS prevention.

\section{Conclusions}

This randomized double-blind placebo control crossover trial demonstrated that the $R$. crenulata extract was not effective in reducing the incidence or severity of AMS. $R$. crenulata extract should not be recommended as a prophylactic for AMS.

\section{Competing interests}

All authors have no competing interests.

\section{Authors' contributions}

TFC is responsible for the study design, performing research, data interpretation and drafting of the article. LLC is responsible for the study design, statistical analysis and drafting of the article. DHS is responsible for revising the article and statistical expertise. $\mathrm{HYL}$ and $\mathrm{CHC}$ are responsible for collection and assembly of data. SHW and WLC both make great contribution to conception and design, critical revision of the article for important intellectual content, interpreting results and revising manuscript. All authors read and approved the final manuscript.

\section{Acknowledgments}

This work was supported by the National Science Council, Taiwan, National Research Program for Biopharmaceuticals Grant (NSC 99-3114-B-182A-002) to T-F Chiu. Study medication and placebo were provided by Kaiser Pharmaceutical \& Biotanico (Tainan, Taiwan). The training camp was provided Army Command Headquarter, Ministry of National Defense R.O.C. (The funders and the pharmaceutical factory had no role in study design, data collection and analysis, decision to publish, or preparation of the manuscript).

\section{Author details}

'Department of Emergency Medicine, Chang Gung Memorial Hospital, Fu-Hsing Street, Kweishan, Taoyuan, Taiwan. ${ }^{2}$ Chang Gung University College of Medicine, Wen-Hua 1st Road, Kweishan, Taoyuan, Taiwan. ${ }^{3}$ Department of Internal Medicine, Far-east Polyclinics, Yong-Sui Street, Taipei, Taiwan. ${ }^{4}$ Division of Biostatistics, Graduate Institute of Epidemiology and Preventive Medicine, College of Public Health, National Taiwan University, Syu-Jhou Road, Taipei, Taiwan. ${ }^{5}$ Department of Family Medicin, Chang Gung Memorial Hospital, Fu-Hsing Street, Kweishan, Taoyuan, Taiwan. ${ }^{6}$ Department of Emergency Medicine, Taipei Medical University Hospital, Wu-Hsing Street, Taipei, Taiwan. ${ }^{7}$ Department of Physical Education, National Taitung University, Chunghua Road, Taitung, Taiwan. ${ }^{8}$ Department of Emergency Medicine, Cathay General Hospital, Sec. 4, Jen-Ai Road, Taipei, Taiwan.

${ }^{9}$ School of Medicine, Fu-Jen Catholic University, Zhongzheng Road, Xinzhuang, Taipei, Taiwan.

Received: 10 April 2013 Accepted: 28 October 2013 Published: 31 October 2013

\section{References}

1. Hackett PH, Roach RC: High-altitude illness. N Engl I Med 2001, 345(2):107-114

2. Imray C, Booth A, Wright A, Bradwell A: Acute altitude illnesses. BMJ 2011 343:d4943.

3. Chow T, Browne V, Heileson HL, Wallace D, Anholm J, Green SM: Ginkgo biloba and acetazolamide prophylaxis for acute mountain sickness: a randomized, placebo-controlled trial. Arch Intern Med 2005, 165(3):296-301.

4. Hackett PH, Rennie D, Levine HD: The incidence, importance, and prophylaxis of acute mountain sickness. Lancet 1976, 2(7996):1149-1155.

5. Seupaul RA, Welch JL, Malka ST, Emmett TW: Pharmacologic prophylaxis for acute mountain sickness: a systematic shortcut review. Ann Emerg Med 2012, 59(4):307-317.

6. Basnyat B, Murdoch DR: High-altitude illness. Lancet 2003, 361(9373):1967-1974.

7. Dumont L, Mardirosoff C, Tramer MR: Efficacy and harm of pharmacological prevention of acute mountain sickness: quantitative systematic review. BMJ 2000, 321(7256):267-272.

8. Basnyat B, Gertsch JH, Holck PS, Johnson EW, Luks AM, Donham BP, Fleischman RJ, Gowder DW, Hawksworth JS, Jensen BT, et al: Acetazolamide $125 \mathrm{mg} \mathrm{BD}$ is not significantly different from $375 \mathrm{mg} \mathrm{BD}$ in the prevention of acute mountain sickness: the prophylactic acetazolamide dosage comparison for efficacy (PACE) trial. High Alt Med Biol 2006, 7(1):17-27.

9. Imray C, Wright A, Subudhi A, Roach R: Acute mountain sickness: pathophysiology, prevention, and treatment. Prog Cardiovasc Dis 2010, 52(6):467-484

10. Panossian A, Wikman G, Sarris J: Rosenroot (Rhodiola rosea): traditional use, chemical composition, pharmacology and clinical efficacy. Phytomedicine 2010, 17(7):481-493.

11. Gertsch JH, Seto TB, Mor J, Onopa J: Ginkgo biloba for the prevention of severe acute mountain sickness (AMS) starting one day before rapid ascent. High Alt Med Biol 2002, 3(1):29-37.

12. Gertsch JH, Basnyat B, Johnson EW, Onopa J, Holck PS: Randomised, double blind, placebo controlled comparison of ginkgo biloba and acetazolamide for prevention of acute mountain sickness among Himalayan trekkers: the prevention of high altitude illness trial (PHAIT). BMJ 2004, 328(7443):797.

13. van Patot MC, Keyes LE, Leadbetter $G$ 3rd, Hackett PH: Ginkgo biloba for prevention of acute mountain sickness: does it work? High Alt Med Biol 2009, 10(1):33-43.

14. Zhang ZJ, Tong Y, Zou J, Chen PJ, Yu DH: Dietary supplement with a combination of Rhodiola crenulata and Ginkgo biloba enhances the endurance performance in healthy volunteers. Chin J Integr Med 2009, 15(3):177-183

15. Abidov M, Crendal F, Grachev S, Seifulla R, Ziegenfuss T: Effect of extracts from Rhodiola rosea and Rhodiola crenulata (Crassulaceae) roots on ATP content in mitochondria of skeletal muscles. Bull Exp Biol Med 2003, 136(6):585-587.

16. Li T, Zhang H: Identification and comparative determination of rhodionin in traditional tibetan medicinal plants of fourteen Rhodiola species by high-performance liquid chromatography-photodiode array detection and electrospray ionization-mass spectrometry. Chem Pharm Bull (Tokyo) 2008, 56(6):807-814. 
17. Salazar H, Swanson J, Mozo K, White AC Jr, Cabada MM: Acute mountain sickness impact among travelers to Cusco. Peru. J Travel Med 2012, 19(4):220-225.

18. De Bock K, Eijnde BO, Ramaekers M, Hespel P: Acute Rhodiola rosea intake can improve endurance exercise performance. Int J Sport Nutr Exerc Metab 2004, 14(3):298-307.

19. Hung SK, Perry R, Ernst E: The effectiveness and efficacy of Rhodiola rosea L.: a systematic review of randomized clinical trials. Phytomedicine 2011, 18(4):235-244

20. Lee SY, Li MH, Shi LS, Chu H, Ho CW, CTC: Rhodiola crenulata extract alleviates hypoxic pulmonary edema in rats. Evid Based Complement Alternat Med 2013, 2013:718739.

21. Swenson ER, Maggiorini M, Mongovin S, Gibbs JSR, Greve I, Mairbäurl H, Bärtsch P: Pathogenesis of high-altitude pulmonary edema: inflammation is not an etiologic factor. JAMA 2002, 287(17):2228-2235.

22. Stream JO, Grissom CK: Update on high-altitude pulmonary edema: pathogenesis, prevention, and treatment. Wilderness Environ Med 2008, 19(4):293-303

23. Icard P, Saumon G: Alveolar sodium and liquid transport in mice. Am J Physiol 1999, 277(6):L1232-L1238.

24. Looney MR, Sartori C, Chakraborty S, James PF, Lingrel JB, Matthay MA: Decreased expression of both the a1-and a2 -subunits of the Na-KATPase reduces maximal alveolar epithelial fluid clearance. Am J Physiol 2005, 289(1):L104-L110.

25. Zhang BC, Li WM, Guo R, Xu YW: Salidroside decreases atherosclerotic plaque formation in low-density lipoprotein receptor-deficient mice. Evid Based Complement Alternat Med 2012, 2012:607508.

26. Sun L, Isaak CK, Zhou Y: Salidroside and Tyrosol from Rhodiola protect H9c2 cell from ischemia/reperfusion-induced apoptosis. Life Sci 2012, 91:151-158.

27. Chen SF, Tsai HJ, Hung TH, Chen CC, Lee CY, Wu CH, Wang PY, Liao NC: Salidroside improves behavioral and histological outcomes and reduces apoptosis via PI3K/Akt signaling after experimental traumatic brain injury. PLoS One 2012, 7(9):e45763.

28. Tu Y, Roberts L, Shetty K, Schneider SS: Rhodiola crenulata induces death and inhibits growth of breast cancer cell lines. J Med Food 2008, 11(3):413-423

29. Lee OH, Kwon Yl, Apostolidis E, Shetty K, Kim YC: Rhodiola-induced inhibition of adipogenesis involves antioxidant enzyme response associated with pentose phosphate pathway. Phytother Res 2011 25(1):106-115

30. Qu ZQ, Zhou Y, Zeng YS, Lin YK, Li Y, Zhong ZQ, Chan WY: Protective effects of a Rhodiola crenulata extract and salidroside on hippocampal neurogenesis against streptozotocin-induced neural injury in the rat. PLoS One 2012, 7(1):e29641.

31. Lee SY, Shi LS, Chu H, Li MH, Ho CW, Lai FY, Huang CY, Chang TC: Rhodiola crenulata and its bioactive components, salidroside and tyrosol, reverse the hypoxia-induced reduction of plasma-membrane-associated $\mathrm{Na}$. KATPase expression via inhibition of ROS-AMPK-PKC \& Pathway. Evid Based Complement Alternat Med 2013, 2013:284150.

32. Wu TY, Ding SQ, Zhang SL, Duan JQ, Li BY, Zhan ZY, Wu QL, Baomu S, Liang BZ, Han SR, Jie YL, Li G, Sun L, Kayser B: Altitude illness in Qinghai-Tibet railroad passengers. High Alt Med Biol 2010, 11(3):189-198.

33. Wang S, Wang FP: [Studies on the chemical components of Rhodiola crenulata]. Yao Xue Xue Bao 1992, 27(2):117-120.

34. Wang S, You XT, Wang FP: [HPLC determination of salidroside in the roots of Rhodiola genus plants]. Yao Xue Xue Bao 1992, 27(11):849-852.

35. Roach RC, Bartsch P, Hackett PH, Oelz O: Hypoxia and Molecular Medicine: Proceedings of the 8th International Hypoxia Symposium: The Lake Louise Acute Mountain Sickness Scoring System. ; 1993.

36. Wang SH, Chen YC, Kao WF, Lin YJ, Chen JC, Chiu TF, Hsu TY, Chen HC, Liu SW: Epidemiology of acute mountain sickness on Jade Mountain, Taiwan: an annual prospective observational study. High Alt Med Biol 2010, 11(1):43-49.

37. Mairer K, Wille M, Bucher T, Burtscher M: Prevalence of acute mountain sickness in the Eastern Alps. High Alt Med Biol 2009, 10(3):239-245.

38. Pesce C, Leal C, Pinto H, González G, Maggiorini M, Schneider M, Bärtsch P: Determinants of acute mountain sickness and success on Mount Aconcagua (6962 m). High Alt Med Biol 2005, 6(2):158-166.
39. Ziaee V, Yunesian M, Ahmadinejad Z, Halabchi F, Kordi R, Alizadeh R, Afsharjoo HR: Acute mountain sickness in Iranian trekkers around Mount Damavand (5671 m) in Iran. Wilderness Environ Med 2003, 14(4):214-219.

40. Gaillard S, Dellasanta P, Loutan L, Kayser B: Awareness, prevalence, medication use, and risk factors of acute mountain sickness in tourists trekking around the Annapurnas in Nepal: a 12-year follow-up. High Alt Med Biol 2004, 5:410-419.

41. Vardy J, Vardy J, Judge K: Can knowledge protect against acute mountain sickness? J Public Health (Oxf) 2005, 27:366-370.

\section{doi:10.1186/1472-6882-13-298}

Cite this article as: Chiu et al:: Rhodiola crenulata extract for prevention of acute mountain sickness: a randomized, double-blind, placebocontrolled, crossover trial. BMC Complementary and Alternative Medicine $201313: 298$

\section{Submit your next manuscript to BioMed Central and take full advantage of:}

- Convenient online submission

- Thorough peer review

- No space constraints or color figure charges

- Immediate publication on acceptance

- Inclusion in PubMed, CAS, Scopus and Google Scholar

- Research which is freely available for redistribution 